\title{
STRetch: detecting and discovering pathogenic short tandem repeat expansions
}

\author{
Harriet Dashnow ${ }^{1,2}$, Monkol Lek ${ }^{3,4}$, Belinda Phipson ${ }^{1}$, Andreas Halman ${ }^{1,5}$, Simon Sadedin ${ }^{1}$, Andrew Lonsdale ${ }^{1}$, \\ Mark Davis ${ }^{6}$, Phillipa Lamont ${ }^{7}$, Joshua S. Clayton ${ }^{8}$, Nigel G. Laing ${ }^{8}$, Daniel G. MacArthur ${ }^{3,4}$ and Alicia Oshlack ${ }^{1,2^{*}}$ (D)
}

\begin{abstract}
Short tandem repeat (STR) expansions have been identified as the causal DNA mutation in dozens of Mendelian diseases. Most existing tools for detecting STR variation with short reads do so within the read length and so are unable to detect the majority of pathogenic expansions. Here we present STRetch, a new genome-wide method to scan for STR expansions at all loci across the human genome. We demonstrate the use of STRetch for detecting STR expansions using short-read whole-genome sequencing data at known pathogenic loci as well as novel STR loci.

STRetch is open source software, available from github.com/Oshlack/STRetch.
\end{abstract}

\section{Background}

Short tandem repeats (STRs), also known as microsatellites, are a set of short (1-6 bp) DNA sequences repeated consecutively. Approximately $3 \%$ of the human genome consists of STRs [1]. These loci are prone to frequent mutations and high polymorphism, with mutation rates $10-100,000$ times higher than average rates throughout the genome [2]. Dozens of neurological and developmental disorders have been attributed to STR expansions [3]. STRs have also been associated with a range of functions such as DNA replication and repair, chromatin organization, and regulation of gene expression $[2,4,5]$.

STR expansions have been identified as the causal DNA mutation in almost 30 Mendelian human diseases [6]. Many of these conditions affect the nervous system, including Huntington's disease, spinocerebellar ataxias, spinal-bulbar muscular atrophy, Friedreich's ataxia, fragile X syndrome, and polyalanine disorders [7]. Most tandem repeat expansion disorders show dominant inheritance, with disease mechanisms varying from expansion of a peptide repeat and subsequent disruption

\footnotetext{
* Correspondence: alicia.oshlack@mcri.edu.au

${ }^{1}$ Murdoch Children's Research Institute, Royal Children's Hospital, Parkville, VIC, Australia

${ }^{2}$ School of Biosciences, The University of Melbourne, Parkville, VIC, Australia Full list of author information is available at the end of the article
}

of protein function or stability, to aberrant regulation of gene expression [8].

STR expansion diseases typically show genetic anticipation, characterized by greater severity and earlier age of onset as the tandem repeat expands through the generations [9]. In many STR diseases, the probability that a given individual is affected increases with the repeat length. In some cases severity also depends on the gender of the parent who transmitted the repeat expansion [10]. The number and position of imperfect repeat units also influences the stability of the allele through generations [9]. Together, these features can be used to identify patients with a disease of unknown genetic basis that might be caused by an STR expansion.

Historically, STRs have been genotyped using polymerase chain reaction (PCR) and gel electrophoresis. In such cases, PCR is performed using primers complementary to unique sequences flanking the STR. The PCR product is then run on a capillary electrophoresis gel to determine its size. Although this method has been scaled to handle dozens of samples, it is still labor-intensive and costly. Each new STR locus to be genotyped requires the design and testing of a new set of PCR primers, along with control samples.

A number of diseases are known to be caused by any one of multiple variants, including STR expansions, single nucleotide variants (SNVs), or short indels. For 
example, there are $>10$ STR loci in as many genes that are known to cause ataxia [11], as well as SNVs and indels in dozens of genes [12]. For such diseases, this can mean hundreds of dollars spent per STR locus, plus additional costs for SNV and short indel testing. For such conditions, there is a clear need for a single genomic test that can detect all relevant disease variants including SNVs, indels, and STRs.

The ability to genotype STRs directly from next-generation sequencing (NGS) data has the potential to reduce both the time and cost to reaching diagnosis and to discover new causal STR loci. It is becoming increasingly common to sequence the genomes or exomes of patients with undiagnosed genetic disorders. Currently, the analysis of these data is focused on SNVs and short indels, and while NGS has identified hundreds of new disease-causing genes, to our knowledge no new pathogenic STR expansions have been discovered. STRs are generally only investigated in an ad-hoc manner at known loci if they are a common cause of the clinical phenotype. The ability to screen for STR expansions in NGS data gives the potential to perform disease variant discovery in those patients for which no known pathogenic variants are found.

The vast majority of current STR genotyping tools for short-read sequencing data (most notably LobSTR [13], HipSTR [14], and RepeatSeq [15]) are designed to look at normal population variation by looking for insertions and deletions within reads that completely span the STR. These tools are limited to genotyping alleles that are less than the read length and require sufficient unique flanking sequence to allow them to be mapped correctly. However, for most STR loci causing Mendelian disease in humans, pathogenic alleles typically exceed $100 \mathrm{bp}$, with pathogenic alleles at some loci in the range of 1000-10,000 bp [16], far exceeding the size cut-off for detection using these algorithms.

One STR genotyper, STRViper [17], which is also designed to detect population variation, has the potential to detect alleles exceeding the read length by looking for shifts in the distribution of insert size from paired reads. This method requires that the insert size distribution has a relatively low standard deviation (SD) and is limited to repeats smaller than the insert size with enough flanking sequence to map both pairs to the reference genome. The mean insert size can be as low as 300$400 \mathrm{bp}$, meaning that for many large pathogenic expansions, there may be very few or no spanning read-pairs. Such methods would therefore have limited utility for the detection of allele sizes expected for pathogenic expansions. Another tool, ExpansionHunter [18], uses read-pair information and recovery of mis-mapped reads to estimate the length of STRs. For known pathogenic sites, ExpansionHunter can be used to determine if the length of the STR is in the pathogenic range. This tool was originally developed for the FTDALS1 repeat and only works on a specific set of pre-defined loci and is therefore not a genome-wide method. Similarly, exSTRa [19] detects expansions in a set of 21 pre-defined loci and requires a set of matched control samples in order to define the statistical probability of an expansion. In contrast to ExpansionHunter, the exSTRa method does not attempt to estimate allele lengths.

While long-read sequencing technologies can potentially sequence through larger repeat loci [20], they are currently far too expensive for clinical use. High error rates and low throughput also make these technologies less suitable for genotyping SNVs and short indels and are thus a poor alternative to short-read sequencing in a clinical setting. Clearly, there is still a great need to be able to detect STR expansions from short read data.

Here we present STRetch, a new method to detect rare expansions at every STR locus in the genome and estimate their approximate size directly from short-read sequencing. We show that STRetch can detect pathogenic STR expansions in short-read PCR-free whole-genome sequencing (WGS) data and can detect expansions at STR loci not known to be pathogenic. We also demonstrate the application of STRetch to solve cases of patients with undiagnosed disease, in which STR expansions are a likely cause.

STRetch is open source software, available from github.com/Oshlack/STRetch.

\section{Results}

\section{The STRetch method}

The STRetch method has been designed to identify expanded STRs from short-read sequencing data and give approximate sizes for these alleles. Briefly, the idea behind STRetch is to first construct a set of additional sequences comprising all possible STR repeat units in the range of 1-6 bp. These are then added to the reference genome as "STR decoy chromosomes." By mapping to this modified genome, STRetch identifies reads that originated from large STR expansions, containing mostly STR sequence, that now preferentially map to the STR decoys. These reads are then allocated back to the genomic STR locus using read-pair information and the locus is assessed for an expansion using a statistical test based on coverage of the STR. A summary of the STRetch method is presented in Fig. 1, with further specifics detailed below.

\section{STR decoy chromosomes: generating an STR-aware reference genome}

A key feature of STRetch is the generation of STR decoy chromosomes to produce a custom STR-aware reference genome. Most aligners have difficulty accurately mapping reads containing long STRs. For example, although 


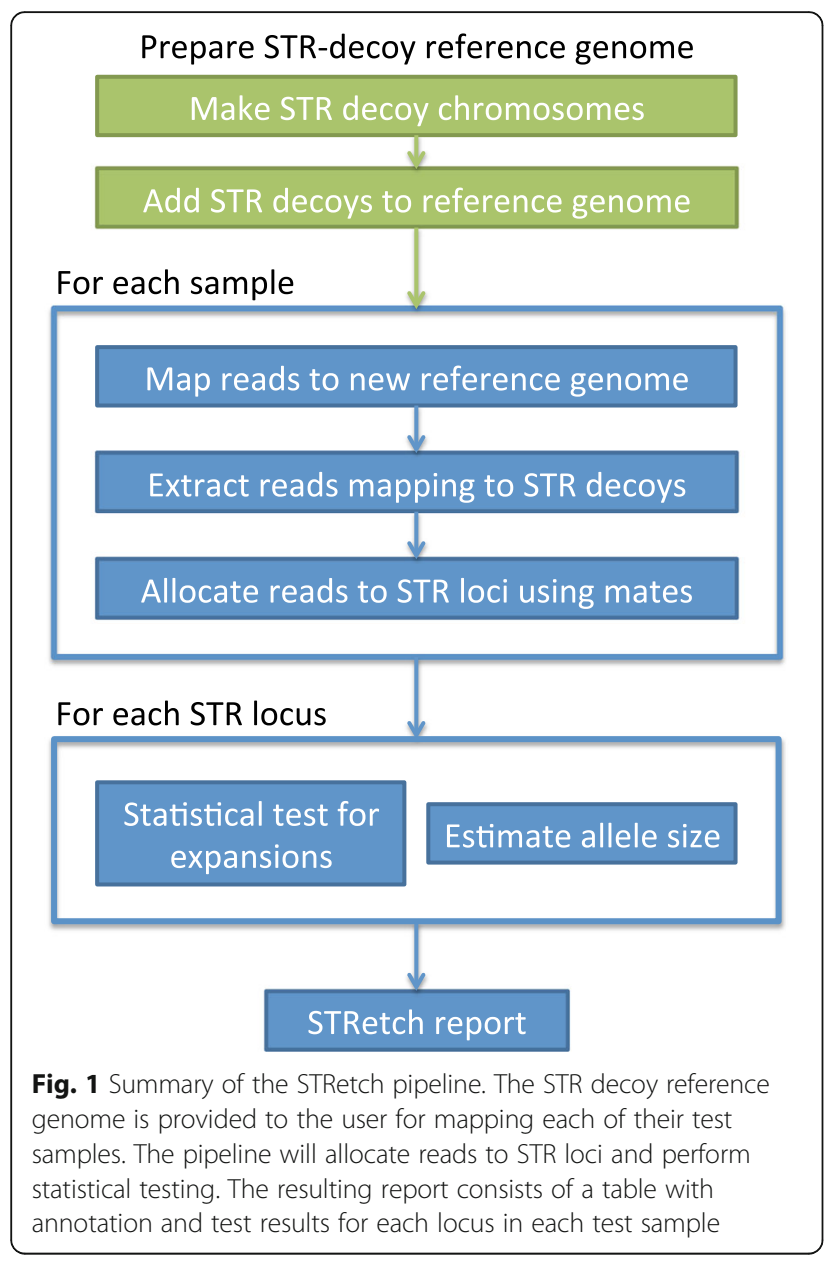

the BWA-MEM algorithm has superior performance for mapping reads containing STRs [21], reads containing long STRs sometimes map to other STR loci with the same repeat unit or completely fail to map [18]. The systematic mis-mapping of STR reads is unsurprising considering that BWA-MEM is optimized to find the longest exact match [22]. For a read made up primarily of STR sequence, the best match is likely to be the longest STR locus in the reference genome with the same repeat unit.

STRetch takes the issue of systematically mis-mapped or unmapped STR reads and uses this as a way to identify reads that contain long STR sequence. To achieve this, we introduce the concept of STR decoy chromosomes. These are sequences that consist of $2000 \mathrm{bp}$ of pure STR repeat units that can be added to any reference genome as additional chromosomes. STR decoy chromosomes for all possible STR repeat units in the range of 1-6 bp are generated and filtered for redundancy, resulting in 501 new chromosomes that are added to the reference genome (STRetch provides hg19 with STR decoys, see "Methods"). While reads with STR lengths similar to the allele length in the reference genome will map to their original locus, reads containing large STR expansions will preferentially align to the STR decoy chromosomes. These reads are then further examined for evidence of a pathogenic expansion.

\section{Mapping to STR decoys to identify reads containing STRs}

Once the new STR decoy reference genome is created, the first step maps reads against the new reference genome using BWA-MEM. If the data have already been mapped, STRetch can optionally extract and re-map a subset of reads likely to contain STRs. Extracted reads are those that aligned to known STR loci (defined using Tandem Repeats Finder (TRF) [23]), as well as any unmapped reads (see "Methods"). Any reads mapping to the STR decoy chromosomes are inferred to have originated from an STR. Typically $\sim 0.01 \%$ of reads map to the STR decoy chromosomes in a PCR-free whole genome.

\section{Determining the origin of STR reads}

Next, the reads that map to the STR decoys are assigned to genomic STR positions. STRetch uses the mapping position of the read at the other end of the DNA fragment (the mate read) to infer from which STR locus each read originates. Known STR loci are obtained from a TRF annotation of the reference genome. For a given read, if the mate maps within $500 \mathrm{bp}$ of a known STR locus with the same repeat unit, then the read is assigned to that locus (or the closest matching locus if multiple loci are present). Only $0.93 \%$ of STR loci are within $500 \mathrm{bp}$ of another STR locus with the same repeat unit (Additional file 1: Figure S1). This distance accounts for the fragment length of the majority of reads (Additional file 1: Figure S2).

After all possible reads are assigned, there may be a difference between the number of reads mapping to a given STR decoy chromosome and the number of reads assigned to all STR loci with that same repeat unit. Unassigned STR reads can occur for a variety of reasons; for example, if their mate also maps to the STR decoy chromosome, if their mate is unmapped, or if their mate does not map in close proximity to a known locus. The number of unassigned reads will increase in samples with very large STR expansions because more read-pairs will originate purely from the STR. This may result in STRetch underestimating the size of very large alleles; however, such loci will still be reported as significant as they are still assigned substantially more reads compared with control samples.

\section{Detecting outlier STR loci}

STRetch next uses a statistical test to identify loci where an individual has an unusually large STR. Specifically, STRetch compares the number of STR decoy reads 
assigned to each locus for a test sample with STR reads from a set of control samples. At each locus the read counts are normalized by dividing by the average coverage of the sample. The set of control samples provide a median and variance of counts for each locus. A statistically robust z-score ("outlier score") is then used to test if the log-normalized number of reads in the test sample is an outlier compared to controls (see "Methods"). The final result is a multiple-testing-adjusted $p$ value describing the significance of an expansion at each locus relative to control samples. Every locus for which reads have been assigned is given a $p$ value.

A variety of control samples can be used in the statistical testing. First, STRetch can be run on a set of controls and the median and variance of the coverage parameters for each locus estimated. These control parameters are then used in testing for significant expansions in disease samples. This approach is ideal for researchers who have access to large sets of sequenced controls. Second, a set of samples that are all being tested can be used as controls for each other in a similar way to the above. The assumption here is that only a small proportion of samples $(<50 \%)$ will have the same expanded locus (we refer to this approach as internal controls). Third, STRetch supplies median and variance parameters estimated from a reference set of PCR-free whole genomes (see "STRetch reveals STR expansions in 97 whole genomes"). This third approach is useful for researchers who only have a limited number of samples to test. The advantage of the first and second options is that the sequencing is usually run at the same center with the same library preparation protocols. However, the datasets may be smaller and therefore provide less robust statistical measures. In most cases, option three (using the supplied parameters from PCR-free whole genomes) is the easiest and most accurate when using relatively small datasets (see Table 1).

\section{Estimating the size of STR alleles}

When scanning the genome for sites of significant expansions, the statistical test is the most important screen. However, for known disease loci the literature has focused on the length of the variant that is associated with pathogenicity. Therefore, STRetch also makes estimates of allele length. STRetch works on the assumption that, for a given locus, the number of reads containing the STR repeat unit is proportional to the length of the repeat in the genome being sequenced. This is because increasing the length of the STR allele increases the likelihood that reads from that locus will be sampled. Hence, STRetch estimates the size of any detected expansion using the normalized read counts allocated to that STR locus. Using simulation, we indeed found that the allocated read counts are linearly related to the length. Specifically, we simulated reads from 100 individuals with the genotype $16 \times \mathrm{CAG} / \mathrm{N} \times \mathrm{CAG}$ at the SCA8 locus, where $\mathrm{N}$ was randomly selected in the range of 0-500 (see "Methods"). Our simulated data exhibit a linear relationship between allele size and the number of reads mapping to the STR decoy chromosome (Additional file 1: Figure S3). We use the slope and offsets from these simulated expansions in estimating the allele size from the normalized coverage at a locus.

\section{Output files}

On completion, STRetch generates a tab-delimited output file for each sample that contains all STR loci for which STR decoy reads were detected. Further information includes $p$ values for statistical significance of an expansion, details of the STR locus (position, repeat unit, size in reference), robust outlier z-score, locus read count, and the allele length estimate. By default, this file is sorted such that the most significant expansions are ranked at the top.

\section{STRetch is able to recover true pathogenic expansions}

In order to test STRetch, we generated PCR-free WGS on ten individuals: nine with known pathogenic STR expansions and one unaffected family member. Samples were sequenced to a mean coverage of $41.74 \times$ (range 38.35-49.57x), then processed using the Broad GATK pipeline (mapped to hg38 with BWA-MEM, then processed using the GATK best practices).

For analysis with STRetch, we first extracted reads overlapping all known STR loci annotated by TRF (see "Methods") and then processed these reads through the STRetch pipeline, using the hg19 reference genome. The STRetch statistics were calculated twice; first using only these ten samples as controls for each other ("internal control") and then using the 97 WGS samples described below as controls ("reference control"). We also ran LobSTR/HipSTR to estimate the size of the short allele in each case. On average, STRetch reported 18 significant STR expansions per sample (range 4-33).

For six of the ten samples we had information about the disease and the estimated allele size by PCR. For the other four samples (Samples 7, 8, 9, and 10), we were initially completely blinded to all patient information, including phenotype. Disease and allele size estimates were only revealed to us after we had correctly identified the causal STR expansion in each case. Table 1 summarizes the results of this analysis, while Fig. 2 and Additional file 1: Table S1 show allele size estimates for these samples using STRetch, LobSTR, HipSTR, and ExpansionHunter.

For the six samples with known information, STRetch correctly identified three true-positive expansions. 


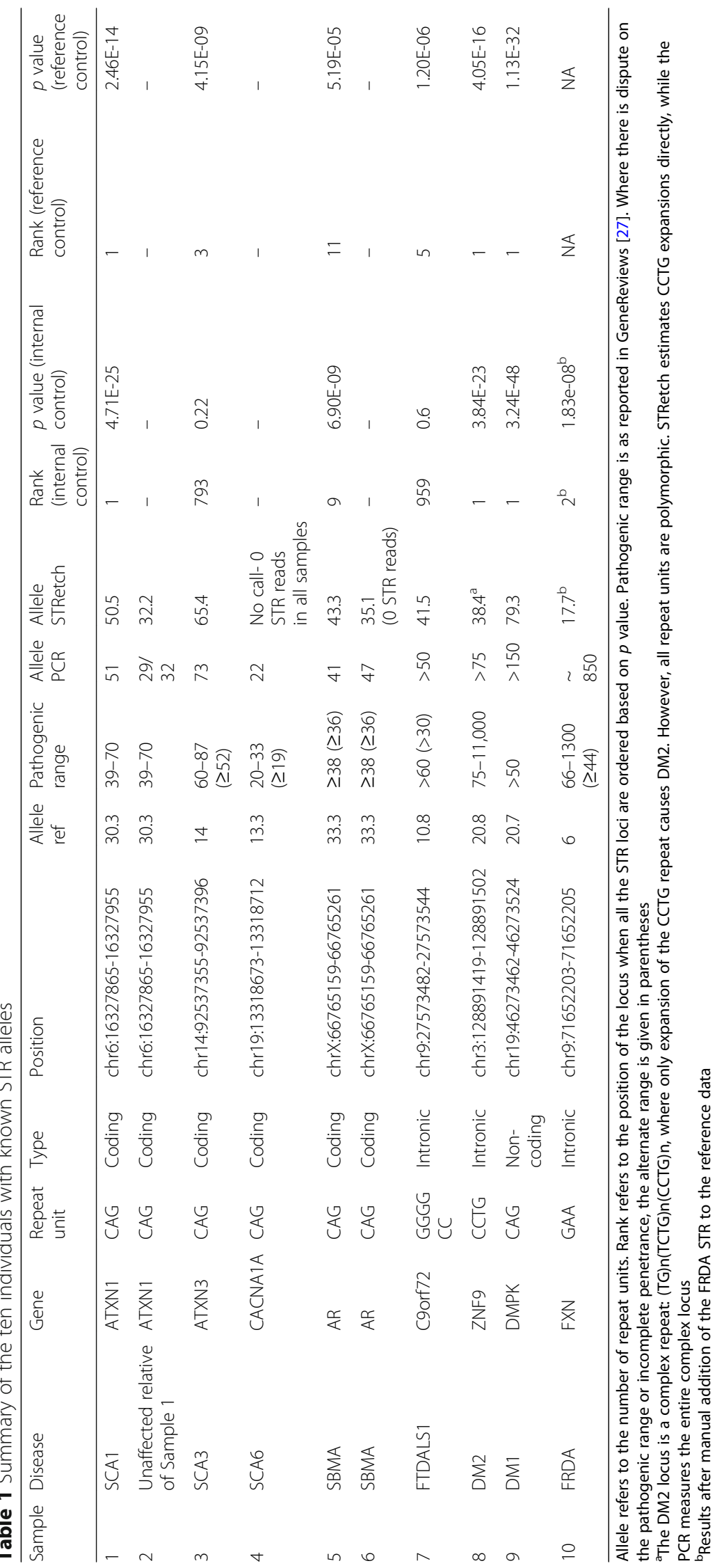




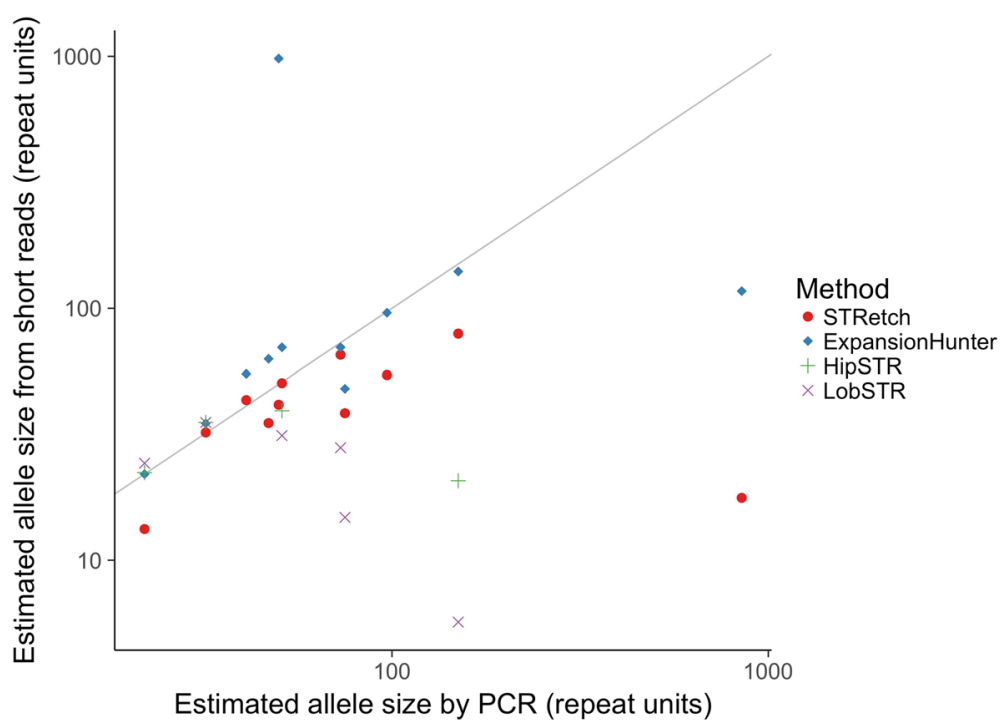

Fig. 2 Relationship between allele sizes estimated by PCR and those called by STRetch, ExpansionHunter, HipSTR, and LobSTR for the true-positive samples. The raw data are available in Additional file 1: Table S1

Furthermore, STRetch correctly failed to detect an expansion in a pathogenic locus in the true negative (Sample 2). STRetch failed to identify the causal locus in two cases (Samples 4 and 6). For Sample 4, the PCR allele length is only 26 bp larger than the reference, making the entire allele $66 \mathrm{bp}$, which is well within the read length of $150 \mathrm{bp}$. STRetch only detects STR expansions that are sufficiently large so that the repeat maps to the STR decoys instead of the reference locus. Indeed, for Sample 4, we see three reads mapped to the genomic locus with a 27-bp insertion and no reads from this locus mapping to the STR decoy. This allele can be detected by tools that look for indels within the read, and indeed both LobSTR and HipSTR are able to correctly call this expansion (Additional file 1: Table S1). Sample 6 was found to have lower coverage over the STR region compared with other samples. However, some evidence of the repeat was observed, such as reads ending in the STR with more repeat units than the reference. As such, this expansion may be detected in future iterations of the software.

For the blinded samples (Samples 7, 8, and 9), we were able to correctly determine the causal STR locus simply by ranking variants by their $p$ values and then looking for any known pathogenic STR loci with a significant $p$ value (Table 1).

For Sample 10, we were initially unable to identify a significant expansion in a known pathogenic gene. After the variant was revealed to be a large GAA expansion at the FRDA locus, we investigated further and discovered that although the reference genome has $6 \times \mathrm{GAA}$ at this position, the STR is missing from the TRF genome annotation. TRF failed to annotate this STR due to its relatively small size. After manually adding this locus to the genome annotation and rerunning the analysis on the ten samples in Table 1, STRetch was able to correctly detect a significant expansion at this locus. Note that this locus was not annotated in the control samples so only an "internal control" analysis was done.

Most of the true positives that were detected had significant expansions when using both the 97 reference controls and the ten internal controls. However, there were two samples (Samples 3 and 7) that were only significant when using the much larger set of reference controls. Therefore, for this size dataset, the use of reference controls generally provides more power.

We compared the PCR-sized expansion in these ten samples with the STRetch length estimates and found that STRetch has about a 20\% standard error. We also found that for very long alleles, STRetch substantially underestimates the allele size. One likely explanation is that the current implementation of STRetch is limited by the insert size of the sequencing data. Some alleles will be so large (e.g. DM1 in Sample 9 is $>450$ bp) that there will be read-pairs where both are completely contained within the STR. In such cases, both pairs will map to the STR decoy and will not be assigned to an STR locus, leading to underestimation of the read length (Additional file 1: Figure S4). In comparison, ExpansionHunter more closely estimated alleles in most cases, with a tendency to overestimate allele sizes (Additional file 1: Table S1). As expected, both LobSTR and HipSTR dramatically underestimated large alleles or did not make a call in many cases.

STRViper was also run on the true-positive samples and reported no significant expansions across all STR 
loci annotated with TRF. This is likely due to the large SD of insert size for these samples (130-150 bp), which is typical for the current standard Illumina protocol. STRViper requires smaller insert sizes of approximately 30 bp or less.

We next assessed the sensitivity, specificity, and false discovery rate (FDR) of STRetch on these samples. We considered STRetch calls in 18 samples for which we had DNA available in our lab (the ten test samples described above and eight samples from the 97 controls described below) on a set of 22 pathogenic loci (Additional file 1: Table S2). This gave a test set of 396 loci. We assumed that any significant STRetch calls $(p<0.05$ after multiple testing correction), beyond the true positive loci described above, are false positives and the two loci that STRetch failed to detect above are false negatives. Any loci where STRetch does not make a significant call are assumed to be true negatives. Of these true negatives, 64 were confirmed by PCR from standard diagnostic testing. Using these assumptions, our measured sensitivity was 0.778 , specificity was 0.974, and the FDR was 0.025. We also ran ExpansionHunter on these samples and found that three of the STRetch calls we had assumed to be false positives were also supported as expanded by ExpansionHunter, although not in the pathogenic range. Three of these (the HD expansion and the two SCA3 expansions) were also confirmed as expanded in the non-pathogenic range by diagnostic PCR. Using these updated values, sensitivity was 0.833 , specificity was 0.974, and FDR was 0.018. Six of our ten false-positive calls were in the FTDALS locus and another two in the SCA3 locus (although these were expanded, just not pathogenic). This indicates that some loci may be harder to correctly identify than others. Indeed, the FTDALS1 locus is known to be difficult to analyze due to homology with other loci [18]. Overall, STRetch achieves FDRs well below our nominal value of 0.05 .

\section{STRetch reveals STR expansions in 97 whole genomes}

We performed PCR-free WGS on a set of 97 individuals, most of whom were being investigated for the cause of their Mendelian disease or were immediate family members of such patients. Many of these cases have inherited neuromuscular disorders. Approximately half of these cases have been previously solved, with the causal variant identified as a SNV or indel. The remaining cases are still unsolved. The set also includes four individuals with ataxia. In addition to patient samples and relatives, there are seven unaffected samples including NA12878. Given its enrichment for individuals with Mendelian disease, this control set may contain pathogenic STR expansions. However, we did not expect a large proportion of samples to have the same STR expansion so our assumptions for the statistical tests were highly unlikely to be violated.
All samples had previously been mapped with BWAMEM against hg19. We used STRetch to extract reads from all annotated STR loci, as well as unmapped reads, and re-mapped these against the hg19 STR decoy genome (see "Methods"). We then proceeded with the rest of the STRetch pipeline. The median and SD were recorded for each locus across all individuals for use as a control set for subsequent analyses. This analysis showed that homopolymer loci are the most variable between individuals, showing dramatically higher SDs, followed by STRs with 5, 6, 3, 4, and then 2 bp repeat units 7(Additional file 1: Figure S5).

To assess the frequency of expansions at known pathogenetic STR loci, we filtered the STRetch results to those significant expansions intersecting with a set of 22 known pathogenic STR loci (Additional file 1: Table S3). We observed 29 significant STR expansions in seven pathogenic loci (summarized in Table 2). Although these are significantly expanded compared to the rest of the control set, their pathogenicity is uncertain as the allele size estimates are only approximate and are often well below the defined pathogenic range.

Nonetheless, a number of the STR expansions are potential candidates for follow-up if the sequenced individuals have a relevant phenotype. STRetch detected a large SCA8 expansion in two individuals; one of whom is an ataxia patient (see below). We also detected a DM2 expansion in another individual. All three variants were highly significantly expanded compared to the other control samples $(p=4.2 \mathrm{e}-24,8.2 \mathrm{e}-23$ and $1.5 \mathrm{e}-10$, respectively) and each was ranked as the most significant for that individual. We have referred these variants back to the originating laboratories to determine whether the variants fit the phenotype and can be validated.

STRetch also identified STR expansions in a number of other pathogenic loci in these samples; however, many of these are unlikely to be sufficiently large to cause disease. Two SBMA expansions were on the limit of detection and significance (ranked 109 and 476, $p=0.001$ and 0.04, respectively). We detected SCA36 and FXTAS expansions, which likely reflect sub-clinical variation at

Table 2 Summary of significant expansions in STR disease loci in 97 WGS samples

\begin{tabular}{lll}
\hline Disease & Gene & Number of individuals \\
\hline SCA8 & ATXN8/ATXN8OS & 2 \\
DM2 & ZNF9 & 1 \\
SBMA & AR & 2 \\
SCA36 & NOP56 & 1 \\
FXTAS & FMR1 & 1 \\
SCA3/MJD & ATXN3 & 11 \\
FTDALS1 & C9orf72 & 11 \\
\hline
\end{tabular}


these loci, with size estimates of $13.5 \times$ AGGCCC and $34.6 \times C C G$, respectively. We detected a surprising number of SCA3/MJD_ATXN3 expansions: 11 samples with estimated allele sizes in the range of $30.5-74.3 \times$ AGC. As $\geq 60 \times A G C$ is considered pathogenic, with $\geq 52 \times$ likely showing incomplete penetrance, many of these may be asymptomatic. However, we have observed STRetch to underestimate allele sizes at this locus so these variants could be larger than predicted. We also detected 11 FTDALS1 expansions in the range of 20.9-32.9×CCCC GG, all within the unaffected size range for this locus. Generally affected individuals have $>60$ repeats. Also, smaller allele sizes are associated with later age of onset, with symptoms appearing as late as 80 years. Consequently, these results may indicate variation within the normal range, individuals with pre-mutations, individuals who have not yet shown symptoms, or false positives. ExpansionHunter confirmed all the SCA8, DM2, and FXTAS expansions, as well as one SBMA, one SCA3, and three of the FTDALS1 expansions. It failed to genotype the SCA36 locus.

These likely non-pathogenic variants at known pathogenic loci highlight the ability of STRetch to explore population variation and to better determine the true non-pathogenic range of these known pathogenic loci.

\section{Genetic diagnosis and validation of an ataxia patient}

As noted above, four ataxia patients were included in our 97 WGS cohort. These patients had previously been tested with for most known ataxia STR expansions, including SCA1, SCA2, SCA3, SCA6, SCA12, SCA17, SCA38, and DRPLA (see Controls 1-4 in Additional file 1: Table S2). The whole genome data had also been examined for causative SNVs and indels in candidate ataxia genes using the GATK Best Practices recommendations [24] and copy number variations using BreakDancer [25] and Genome STRiP [26] without success in diagnosis.

In one of these patients (Control 1), STRetch identified the highly significant expansion of SCA8 (noted above), a known but rare disease locus in ATXN8 with an outlier z-score of $11.11(p=3.55 \mathrm{e}-24)$ and an estimated allele size of $54.4 \times$.

As a result, this SCA8 expansion was validated using a PCR assay, which confirmed a pathogenic CAG expansion with an allele size of $97 \times$. In addition, an affected sibling, not sequenced using WGS, was tested for an expansion at the same locus and was similarly determined to have a pathogenic allele of $\sim 96 \times$. The likely pathogenic range for this STR is $80-250$ repeat units, with uncertain pathogenicity in the range of 50-70 repeat units. However, this locus shows incomplete penetrance, with cases of unaffected individuals observed at all allele sizes [27]. It is noteworthy that STRetch directly estimates the size of the STR, while PCR assays at this locus also include the size of the adjacent non-pathogenic but highly polymorphic CTA repeat. As such, this estimate from STRetch should be interpreted as $65 \times$ when comparing to the PCR result.

We also ran LobSTR and HipSTR on the WGS data of this patient. At the expanded pathogenic locus, LobSTR called a homozygous 6xTGC insertion, while HipSTR called a homozygous indel from deletion of one TAC repeat unit upstream of SCA8 and an insertion of seven TGC repeat units, for a net total six repeat unit insertion. Both tools report a reference allele size of $+15 \times$, so the total allele size is $21 \times$ in both cases. PCR analysis of the proband and sibling indicated that both expansions were heterozygous with a short allele size of $\sim 29 x$. However, these sizes may not be directly comparable due to potential variation in the definition of the reference locus size.

\section{STRetch can detect expansions at novel loci}

To our knowledge, STRetch is the only method currently available that can use short-read sequencing to screen the entire genome for rare STR expansions. In order to demonstrate that STRetch can indeed detect expansions at loci not previously associated with disease, we performed validation using orthogonal technologies: PCR and Sanger sequencing and PacBio long-read sequencing.

First, we used PCR to estimate the size of an STR that STRetch called highly significant in Sample 5 . The locus is a 5-base AAACT repeat in an intron of the MTHFD2 gene (chr2:74430970-74,431,055). This was the most significantly expanded locus in this sample with a $p$ value of $4.81 \mathrm{E}-21$ and a predicted size of 37 repeat units. We designed a PCR assay to genotype the size of the allele in this sample as well as five samples that were not predicted to contain this expansion by STRetch (Samples 1, 2, 6, 8, and 9 from above, Additional file 1: Figure S6). A standard control sample of unknown genotype (CEPH individual 1347-02) was also tested. Sanger sequencing yielded an allele size of $>59$ repeat units in Sample 5 (the sequencing quality dropped off beyond this size), significantly larger than the alleles from the control samples $(p=2.71 \mathrm{E}-03$, Additional file 1: Figure S7), as predicted by STRetch. By sizing the PCR product on a gel, we estimate the larger allele in this sample to be 62 repeat units. We configured ExpansionHunter to estimate the size of this locus resulting in a genotype of 47/64, much larger than the length of 17.2 repeats in the genome. While this STR is unlikely to be pathogenic, this result highlights the potential to use STRetch to screen for novel expansions that may be related to disease.

To further demonstrate the utility of STRetch to identify novel loci as expanded (relative to controls), we 
compared our analysis of short-read data with a previously published genome analysis which utilized longread PacBio data [28]. Specifically, we ran STRetch on Illumina short-read data from an artificial diploid sample created by combining the two haploid genomes, $\mathrm{CH} 1$ and $\mathrm{CH} 13$ (two replicates), and called significantly expanded loci compared with our 97 reference controls. We then compared all significant STRetch hits with variants called from long-read PacBio sequencing of the two haploid samples. Of the 18 significant STRetch calls (over two replicates) we were able to confirm 14 from the PacBio data as expanded (Additional file 1: Table S4). Of the four non-validated calls, two were in the non-standard chromosome chrUn_gl000220, which was not present in the PacBio variant data. The remaining two were both homopolymer A STRs. Homopolymers were excluded from the PacBio variant call set, preventing validation of these loci. While none of the loci represent likely pathogenic expansions, this analysis demonstrates that truly significant expanded variants can be found in novel loci using STRetch with a low FDR.

\section{Discussion}

STRetch is currently the only genome-wide method to scan for STR expansions using short-read WGS data. We have demonstrated the ability of STRetch to detect known pathogenic STR expansions in short-read PCR-free WGS data. STRetch correctly detected the pathogenic expansion in most of our ten test samples; seven true positives and one true negative were correctly identified; two expansions were missed. The size of one of the missed expansions was below the detection threshold for STRetch. The method performed well on these samples, with a sensitivity of 0.778 , specificity of 0.974 , and a FDR of 0.025 . We applied STRetch to 97 PCR-free WGS samples to detect both potentially pathogenic STR expansions and expansions of moderate length in STR disease loci, where the allele size is likely below the threshold for disease. Importantly, this set of analyzed genomes can act as a control set and statistical parameters for the STR loci are provided to use in testing for expansions with STRetch. Within this cohort, STRetch revealed a previously undetected pathogenic STR expansion in SCA8 that was validated by PCR in the proband and an affected sibling.

As expected, we found that STR genotypers such as LobSTR and HipSTR, which are designed to genotype short STR variation, were unable to detect large pathogenic variants in WGS data. These tools instead called a homozygous genotype, corresponding to the size of the non-expanded allele, called a heterozygote with slight variation in the small allele, or failed to make a call. Using these tools in conjunction with STRetch allows the estimation of the short allele in cases where STRetch has detected an expansion, allowing us to obtain a more complete picture of the genotype from short-read sequencing data.

ExpansionHunter performed relatively well when estimating allele sizes, although tended to overestimate, where STRetch tended to underestimate. However, a key limitation of ExpansionHunter is that it does not use a statistical basis for detecting significantly expanded loci and is not currently configured to estimate lengths across the genome; each locus of interest must be defined in a separate configuration file. Therefore, this cannot be used as a genome-wide scan for novel loci.

The main limitation of STRetch is its tendency to underestimate the allele size of STR expansions, especially for variants larger than the insert size. However, this limitation is mostly relevant for known pathogenic loci where there is already an established relationship between the length of the allele and disease characteristics. In genome-wide scans for rare expansions, we believe it is more important to use a statistical test that indicates the probability that an expansion exceeds the normal population variation. Estimating allele length by itself does not provide this information. Another limitation of STRetch is that it has not been tested on PCR+ or targeted sequencing data.

We have demonstrated the potential application of STRetch to discover a novel significant expansion at an intronic STR locus in MTHFD2 and validated it by PCR and Sanger sequencing. In addition, further expansions detected by STRetch were also shown to be expanded using long-read sequencing data.

\section{Conclusions}

Here we have introduced STRetch, a method to test for rare STR expansions from WGS data. We have shown that STRetch can detect pathogenic STR expansions relevant to Mendelian disease with a low FDR. Although the emphasis has been on STRs known to cause Mendelian disease, a key advantage of STRetch over other methods is its genome-wide approach. STRetch performs statistical tests for expansions at every STR locus annotated in the reference genome and so has the potential to be used not only for diagnostics, but also in research to discover new disease-associated STR expansions. We hope the application of STRetch to WGS of patient cohorts will enable new discoveries of disease-causing STR expansions.

\section{Methods}

\section{The STRetch pipeline}

The STRetch pipeline is implemented using the Bpipe [29] pipeline framework (v0.9.9.3). This allows for a pipeline combining standard bioinformatics tools with novel scripts and is compatible with many 
high-performance computing environments, allowing large-scale parallelization over multiple samples (see Additional file 1: Supplementary Methods).

To summarize the pipeline and components:

Reads are mapped to the reference genome with STR decoy chromosomes using BWA-MEM [22] (v0.7.12) and SAMtools [30] (v1.3.1). STRetch then counts the number of reads mapping to each STR decoy chromosome using bedtools [31] (v2.26.0). Reads mapping to the STR decoy chromosomes are allocated to an STR locus using paired information (Python v3.5.2 script: identify_locus.py). Median coverage over the whole genome or exome target region is calculated using goleft covmed [32] (v0.1.8), which is later used to normalize the counts. STRetch then predicts the size of the expansion using the number of reads allocated to the locus (Python script: estimateSTR.py).

The STRetch pipeline is freely available under an MIT license from github.com/Oshlack/STRetch.

\section{Generating STR decoy chromosomes}

To produce STR decoy chromosomes, STRetch generates a set of all possible STR repeat units in the range of 1-6 bp. These are then grouped by those repeat units that are equivalent as a circular permutation of each other or the reverse complement. For each group, the first repeat unit lexicographically was taken to represent that group, for example, $\mathrm{CAG}=\mathrm{AGC}=\mathrm{GCA}=\mathrm{CTG}=\mathrm{TGC}=\mathrm{GCT}$; the group is represented by AGC. STRetch filters out repeat units that could be represented by multiples of a shorter repeat unit. For example, ATAT would be filtered out as it is already represented by AT. This resulted in 501 unique repeat units. STRetch then uses an "STR decoy chromosome" for each repeat unit, which consists of the repeat unit repeated in tandem for 2000 bp (script: decoy_STR.py). This length was chosen to well exceed the insert size, but is configurable. The additional chromosomes can be added to any reference genome (hg19 with STR decoy chromosomes was used for all analyses).

\section{Extracting likely STR read-pairs from aligned BAMs}

In the case where reads have already been mapped to a reference genome, STRetch provides the option of extracting likely STR read-pairs from the BAM file for analysis, rather than remapping all reads in the sample.

In this case, STRetch defines a region where STR reads are likely to align by taking the TRF [23] annotation of the reference genome and expanding the region to include $800 \mathrm{bp}$ of flanking sequence on each side. Reads aligned to this region, and all unmapped reads, are extracted using SAMtools view. These are sorted to place together read-pairs using SAMtools collate and then are extracted in fastq format using bedtools bamtofastq. Unpaired reads are discarded.

\section{Aligning and allocating reads to STR loci}

STRetch uses BWA-MEM to align reads to the custom reference genome. Any read mapping to the STR decoy chromosomes is presumed to have originated from an STR locus.

To determine from which STR locus the reads originated, the mates of the reads mapping to a given STR decoy chromosome are collected. If the mate maps within 500 bp of a known STR locus with the same repeat unit, it is assigned to that locus. If multiple loci fall in this range, the read is assigned to the closest locus. This distance was chosen because the mean insert size of our WGS data was 372-415 bp (Additional file 1: Figure S2), so we expect the mate to map within $500 \mathrm{bp}$ or less of the STR locus. We found that increasing this value did not increase the number of reads allocated to true-positive loci. Another consideration when setting this parameter is the potential to misallocate reads from neighboring STR loci. To combat this, reads are only allocated to a locus with a matching repeat unit. Although rare, there are instances of two STR loci with the same repeat unit occurring close together. In hg19, 0.93\% of STR loci are within 500 bp of another STR locus with the same repeat unit, $1.34 \%$ within $1000 \mathrm{bp}$, and $2.04 \%$ within $2000 \mathrm{bp}$ (see Additional file 1: Figure S1). We used $500 \mathrm{bp}$ as the smallest value of this parameter that allows detection of all relevant reads while minimizing inclusion of inappropriate reads. This value can be configured in the pipeline if required.

To correct for library size (total number of aligned reads), the counts for each STR locus are normalized against the median coverage for that sample. Counts are normalized to a median coverage of $100 \times$ : $\log _{2}(100 \times$ (raw counts +1$)$ /median sample coverage)). $\log _{2}$ normalized counts are used in subsequent statistical analyses.

\section{Detecting outliers}

To detect individuals with unusually large STRs, STRetch calculates an "outlier score" for each individual at each locus. The outlier score is a z-score calculated using robust estimates, with a positive score indicating the STR is larger than the median. Robust estimates are used to reduce the impact of potential expansions present in control samples, which can be particularly important when comparing within a small cohort of samples.

A robust $\mathrm{z}$-score and $p$ value is calculated for each locus, $l$, using the log-normalized counts. First, the median and variance across all samples for locus, $l$, is estimated using Huber's M-estimator [33, 34]. This calculation can be performed over all samples in a batch or a set of control samples (estimates from the set of 97 PCR-free whole genomes are provided with STRetch). We test the null hypothesis that the log counts, $y_{i l}$, at 
locus $l$ for sample $i$ is equal to the median log counts at locus $l$ for the control samples. The alternative hypothesis is that the median log counts for locus $l$ are greater for sample $i$ compared to the control samples. Hence for each sample $i$ and locus $l$ we obtain a robust z-score

$$
z_{i l}=\frac{y_{i l}-M}{\sigma_{M}},
$$

where $M$ is the median and $\sigma_{M}$ is the square root of the M-estimator of the variance. One-sided $p$ values are then obtained from the standard normal distribution and adjusted for multiple testing across the loci using the Benjamini-Hochberg method [35]. A locus is called significant if the adjusted $p$ value is $<0.05$.

\section{Estimating allele sizes}

We reasoned that the size of an expanded allele would be proportional to the number of reads containing STR sequence and hence the number of reads allocated to the STR locus. In order to estimate allele sizes we performed simulations of a single locus at a range of allele sizes. Specifically, reads were simulated from the SCA8 locus in ATXN8. One allele was held constant at 16xAGC and then we simulated repeat lengths in the other allele in the range of $0-500$ repeat units (selected at random from a uniform distribution). Alternate versions of the hg19 reference genome with these alleles were produced using GATK v3.6 FastaAlternateReferenceMaker. Reads were simulated from 10,000 bp either side of the SCA8 locus using ART MountRainier2016-06-05 [36]. Reads were 150-bp paired-end, with nsert sizes sampled from a normal distribution (mean 500 bp, SD 50 bp) and 30× coverage (proportional coverage sampled from each haplotype). The Illumina error profile was used. Simulation code is available at github.com/hdashnow/STR-pipelines.

A plot of the number of reads mapping to the AGC decoy chromosome against the number of AGC repeat units inserted into the ATXN8 locus shows a clear linear relationship between these two variables (Additional file 1: Figure S3).

We can use this information from the simulated data to provide a point estimate of the allele size of any new sample we analyze with STRetch in the following manner. We fit a linear regression between the number of reads mapping to the STR decoy and the size of the allele from the simulated data (both $\log _{2}$ transformed), in order to obtain estimates of the intercept and slope parameters, $\beta_{0}$ and $\beta$,

$$
y=\beta_{0}+\beta x+\varepsilon, \varepsilon \sim N\left(0, \sigma^{2}\right) .
$$

Here $y$ is $\log _{2}$ (coverage) and $x$ is $\log _{2}$ (allele size). Given a new data point from a real sample, the $\log _{2}$ (coverage) for an STR locus of interest, the point estimate of the allele size is thus

$$
\log _{2}(\text { allelesize })=\frac{\log _{2}(\text { coverage })-\hat{\beta}_{0}}{\hat{\beta}} .
$$

where allele size is the number of base pairs inserted relative to the reference and coverage is the normalized number of reads allocated to the locus.

\section{Reference data}

Reference genome: ucsc.hg19.fasta, with STR decoy chromosomes added as described above.

STR positions in genome annotated bed file: hg19.simpleRepeat.txt.gz. Source: http://hgdownload.cse.ucsc.edu/ goldenPath/hg19/database/.

Known STR loci are obtained by performing a TRF [23] annotation of the reference genome. Pre-computed annotations of many genomes are available from the UCSC Table Browser (https://genome.ucsc.edu/cgi-bin/hgTables) [37]. TRF annotations are converted to bed files annotated with two additional columns: the repeat unit/motif and the number of repeat units in the reference.

\section{Running other STR genotypers}

To estimate the size of the shorter allele, LobSTR and HipSTR were run on BAM files containing the locus of interest and $1000 \mathrm{bp}$ of flanking sequence on either side. We used LobSTR version 4.0.6 with default settings and the LobSTR reference genome and annotation hg19_v3.0.2. HipSTR version 0.4 was used --min-reads 2 and otherwise default settings, with the provided hg19 reference genome and annotation. In some cases, the tools make a different call as to the reference allele in hg19. To make variant calls comparable to STRetch calls, we converted genotypes to numbers of repeat units inserted relative to the reference defined by that tool, then applied that to the reference allele given by STRetch. For example, if the STRetch reference allele is 20 repeat units, while in HipSTR it is 10 repeat units, a HipSTR genotype of $10 / 15$ would be reported as 20/25 All imperfect repeat units or other variation were ignored and only the total size of alleles taken.

ExpansionHunter version 2.5.5 was run using the 17 provided STR loci specifications, as well as manually defined loci for STRs in ATXN8, MTHFD2, NOP56, and ZNF9 (see Additional file 1: Supplementary Methods). The "original" version of STRViper was downloaded from http://bioinf.scmb.uq.edu.au/STRViper and run using default settings.

\section{Validation of novel STR expansions}

To validate the novel STR expansion in an intron of MTHFD2 (as predicted by STRetch), PCR was conducted 
using GoTaq G2 colorless master mix (Promega, USA) with $0.5-\mu \mathrm{M}$ primers (see Additional file 1: Table S5) and $10 \mathrm{ng}$ of template DNA per reaction. Cycling was as follows: $95^{\circ} \mathrm{C}$ for $2 \mathrm{~min}, 40$ cycles of $95^{\circ} \mathrm{C}$ for $15 \mathrm{~s}, 68^{\circ} \mathrm{C}$ for $15 \mathrm{~s}$, and $72^{\circ} \mathrm{C}$ for $30 \mathrm{~s}$, and a final extension for $5 \mathrm{~min}$ at $72{ }^{\circ} \mathrm{C}$. Samples were analyzed on a $2 \%$ agarose gel stained with ethidium bromide $(0.5 \mu \mathrm{g} / \mathrm{mL})$. Product sizes and STR length were estimated relative to a 100-bp DNA ladder by generating a standard curve (NEB, USA). For sequencing, individual alleles were separated by band-stab PCR [38] (Additional file 1: Table S5), purified using a QIAquick PCR purification kit (QIAGEN, USA), and sequenced using the Sanger method. The samples tested were Samples 1, 2, 5, 6, 8, and 9 from the true-positive samples described above and one standard control sample 1347-02 (CEPH).

To validate STRetch results with long-read data, STRetch was run on Illumina short-read data from an artificial diploid sample created by combining the two haploid genomes, $\mathrm{CH} 1$ and $\mathrm{CH} 13$. The two replicates were obtained from SRA: https://www.ncbi.nlm.nih.gov/sra, accession numbers ERX1413365 and ERX1413368 [39]. We compared the STRetch results to publicly available PacBio variants for these same samples [28]. The authors generated these variants using their SMRT-SV software [27] against the hg38 reference genome. Long structural variants $(\mathrm{SVs})>50 \mathrm{bp}$ were obtained from dbVar; https://www.ncbi.nlm.nih.gov/dbvar accession number nstd137 [40] and short SVs from http://eichler lab.github.io/pacbio_variant_caller/ [41]. A STRetch call was considered validated if the PacBio data contained a substantial expansion relative to the reference genome at the same position with the same repeat unit.

\section{Additional file}

Additional file 1: Supplementary tables, figures and methods

STRetch_additional_file_1. (PDF $1095 \mathrm{~kb}$ )

\section{Funding}

HD is supported by an Australian Government Research Training Program (RTP) Scholarship, a Murdoch Children's Research Institute Top Up Scholarship, and an Australian Genomics Health Alliance PhD Award, funded by NHMRC (GNT1113531). NGL is supported by NHMRC Fellowship APP1117510, NHMRC Project Grant APP1080587, and EU Collaborative Grant APP1055295. AO is funded by a National Health and Medical Research Council, Career Development Fellowship GNT1126157. This work was supported in part by the National Human Genome Research Institute, the National Eye Institute, and the National Heart, Lung and Blood Institute grant UM1 HG008900.

\section{Availability of data and materials}

The STRetch software is available from https://github.com/Oshlack/STRetch [42]. Control sample summary statistics are available from Figshare (https:// doi.org/10.6084/m9.figshare.6830282) [43]. PCR-free WGS of the ten test samples is available from the Sequence Read Archive (https://www.ncbi.nlm.nih.gov/ sra), accession SRP148723 (individual sample accessions SRX4114164SRX4114173). Public short-read data used for validation was obtained from SRA, accession numbers ERX1413365 and ERX1413368 [39]. Corresponding validated long SVs were obtained from dbVar (https://www.ncbi.nlm. nih.gov/dbvar) accession number nstd137 [40] and short SVs from http:// eichlerlab.github.io/pacbio_variant_caller/ [41].

\section{Authors' contributions}

$\mathrm{HD}$ and $\mathrm{AO}$ conceived the method and drafted the manuscript. HD implemented the algorithm and performed data analysis. BP and SS contributed to algorithm development. AH performed data analysis. $H D$, AH, SS, and AL performed software testing. MD, NGL, and JSC performed experimental validation. NGL and PL contributed patient samples. ML and DGM provided reference samples. DGM provided sequencing and computational resources. ML, BP, AH, AL, MD, JSC, NGL, and DGM contributed to the manuscript. All authors read and approved the final manuscript.

\section{Ethics approval and consent to participate}

This study was approved by the University of Western Australia Human Research Ethics Committee (IRB approval number RA/4/20/1008). All individuals have given written informed consent for publication. The experimental methods in this study comply with the Helsinki Declaration.

Competing interests

The authors declare that they have no competing interests.

\section{Publisher's Note}

Springer Nature remains neutral with regard to jurisdictional claims in published maps and institutional affiliations.

\section{Author details}

${ }^{1}$ Murdoch Children's Research Institute, Royal Children's Hospital, Parkville, VIC, Australia. ${ }^{2}$ School of Biosciences, The University of Melbourne, Parkville, VIC, Australia. ${ }^{3}$ Analytic and Translational Genetics Unit, Massachusetts General Hospital, Boston, MA, USA. ${ }^{4}$ Program in Medical and Population Genetics, Broad Institute of MIT and Harvard, Cambridge, MA, USA. ${ }^{5}$ Florey Institute of Neuroscience and Mental Health, University of Melbourne, Parkville, VIC, Australia. ${ }^{6}$ Department of Diagnostic Genomics, PathWest Laboratory Medicine, QEIl Medical Centre, Nedlands, WA, Australia. ${ }^{7}$ Neurogenetic Unit, Royal Perth Hospital, Perth, WA, Australia. ${ }^{8}$ Harry Perkins Institute of Medical Research, Centre for Medical Research, University of Western Australia, Nedlands, WA, Australia.

Received: 18 December 2017 Accepted: 7 August 2018

Published online: 21 August 2018

\section{References}

1. The 1000 Genomes Project Consortium, Abecasis GR, Altshuler D, Auton A, Brooks LD, Durbin RM, et al. A map of human genome variation from population-scale sequencing. Nature. 2010;467:1061-73.

2. Gemayel R, Vinces MD, Legendre M, Verstrepen $\mathrm{KJ}$. Variable tandem repeats accelerate evolution of coding and regulatory sequences. Annu Rev Genet. 2010:44:445-77.

3. Orr HT, Zoghbi HY. Trinucleotide repeat disorders. Annu Rev Neurosci. 2007; 30:575-621.

4. Hamada H, Seidman M, Howard BH, Gorman CM. Enhanced gene expression by the poly (dT-dG). poly (dC-dA) sequence. Mol Cell Biol. 1984;4:2622-30.

5. Li YY-CC, Korol AB, Fahima T, Beiles A, Nevo E. Microsatellites: genomic distribution, putative functions and mutational mechanisms: a review. Mol Ecol. 2002:11:2453-65.

6. Gatchel JR, Zoghbi HY. Diseases of unstable repeat expansion: mechanisms and common principles. Nat Rev Genet. 2005;6:743-55.

7. van Eyk CL, Richards RI. Dynamic mutations. Tandem repeat polymorphisms New York: Springer; 2012. p. 55-77.

8. Hannan AJ, editor. Tandem repeat polymorphisms: genetic plasticity, neural diversity and disease. Austin/New York: Landes Bioscience/Springer Science +Business Media; 2012

9. Mirkin SM. Expandable DNA repeats and human disease. Nature. 2007;447: $932-40$

10. Sherman SL, Jacobs PA, Morton NE, Froster-Iskenius U, Howard-Peebles PN, Nielsen $\mathrm{KB}$, et al. Further segregation analysis of the fragile $\mathrm{X}$ syndrome with special reference to transmitting males. Hum Genet. 1985;69:289-99. 
11. Margolis RL. The spinocerebellar ataxias: order emerges from chaos. Curr Neurol Neurosci Rep. 2002;2:447-56.

12. Fogel BL, Lee H, Deignan JL, Strom P, Kantarci S, Wang X, et al. Exome sequencing in the clinical diagnosis of sporadic or familial cerebellar ataxia. JAMA Neurol. 2014;71:1237-46

13. Gymrek M, Golan D, Rosset S, Erlich Y. lobSTR: A short tandem repeat profiler for personal genomes. Genome Res. 2012;22:1154-62.

14. Willems T, Zielinski D, Gordon A, Gymrek M, Erlich Y. Genome-wide profiling of heritable and de novo STR variations. Nat Methods. 2017;14:590-2.

15. Highnam G, Franck C, Martin A, Stephens C, Puthige A, Mittelman D. Accurate human microsatellite genotypes from high-throughput resequencing data using informed error profiles. Nucleic Acids Res. 2013;41:e32.

16. Ashley EA. Towards precision medicine. Nat Rev Genet. 2016;17:507-22.

17. Cao MD, Tasker E, Willadsen K, Imelfort M, Vishwanathan S, Sureshkumar S, et al. Inferring short tandem repeat variation from paired-end short reads. Nucleic Acids Res. 2014;42:e16.

18. Dolzhenko E, van Vugt JJFA, Shaw RJ, Bekritsky MA, van Blitterswijk M, Kingsbury $Z$, et al. Detection of long repeat expansions from PCR-free whole-genome sequence data. bioRxiv. 2016; http://biorxiv.org/content/ early/2016/12/19/093831.abstract

19. Tankard RM, Delatycki MB, Lockhart PJ, Bahlo M. Detecting known repeat expansions with standard protocol next generation sequencing, towards developing a single screening test for neurological repeat expansion disorders. bioRxiv. 2017; http://biorxiv.org/content/early/2017/06/30/157792.abstract

20. Chaisson MJP, Huddleston J, Dennis MY, Sudmant PH, Malig M, Hormozdiari $F$, et al. Resolving the complexity of the human genome using singlemolecule sequencing. Nature. 2015;517:608-11.

21. Gymrek M. A genomic view of short tandem repeats. Curr Opin Genet Dev. 2017:44:9-16.

22. Li H. Aligning sequence reads, clone sequences and assembly contigs with BWA-MEM. arXiv Prepr arXiv. 2013:3. http://arxiv.org/abs/1303.3997

23. Benson G. Tandem repeats finder: a program to analyze DNA sequences. Nucleic Acids Res. 1999;27:573.

24. Van der Auwera GA, Carneiro MO, Hartl C, Poplin R, del Angel G, LevyMoonshine A, et al. From FastQ data to high-confidence variant calls: the Genome Analysis Toolkit best practices pipeline. Curr Protoc Bioinformatics. 2013:43:11.10.1-33.

25. Chen K, Wallis JW, McLellan MD, Larson DE, Kalicki JM, Pohl CS, et al, BreakDancer: an algorithm for high-resolution mapping of genomic structural variation. Nat Methods. 2009;6:677-81.

26. Handsaker RE, Van Doren V, Berman JR, Genovese G, Kashin S, Boettger LM, et al. Large multiallelic copy number variations in humans. Nat Genet. 2015; 47:296-303.

27. Adam MP, Ardinger HH, Pagon RA, Wallace SE, Bean LJ, Stephens K, et al. GeneReviews. Seattle, WA: University of Washington; 2018.

28. Huddleston J, Chaisson MJ, Meltz Steinberg K, Warren W, Hoekzema K, Gordon DS, et al. Discovery and genotyping of structural variation from long-read haploid genome sequence data. Genome Res. 2017;27:677-85.

29. Sadedin SP, Pope B, Oshlack A. Bpipe: a tool for running and managing bioinformatics pipelines. Bioinformatics. 2012;28:1525-6.

30. Li H, Handsaker B, Wysoker A, Fennell T, Ruan J, Homer N, et al. The sequence alignment/map format and SAMtools. Bioinformatics. 2009;25: 2078-9.

31. Quinlan AR, Hall IM. BEDTools: a flexible suite of utilities for comparing genomic features. Bioinformatics. 2010;26:841-2.

32. Pedersen B. goleft. 2016. github.com/brentp/goleft

33. Ripley BD. Modern applied statistics with S. New York: Springer; 2002

34. Huber PJ. Robust statistics. New York: Wiley; 1981.

35. Benjamini $Y$, Hochberg $Y$. Controlling the false discovery rate: a practical and powerful approach to multiple testing. J R Stat Soc Ser B JSTOR. 1995; 57:289-300.

36. Huang W, Li L, Myers JR, Marth GT. ART: a next-generation sequencing read simulator. Bioinformatics. 2012;28:593-4

37. Karolchik D, Hinrichs AS, Furey TS, Roskin KM, Sugnet CW, Haussler D, et al. The UCSC table browser data retrieval tool. Nucleic Acids Res. 2004;32: D493-6.

38. Bjourson AJ, Cooper JE. Band-stab PCR: a simple technique for the purification of individual PCR products. Nucleic Acids Res. 1992;20:4675.

39. Eichler $\mathrm{E}$, Surti U. Evaluating variant calling accuracy with $\mathrm{CHM} 1$ and $\mathrm{CHM} 13$ haploid data. Accession PRJEB13208. BioProject. 2016. https://www.ncbi.nlm. nih.gov/bioproject/316945.
40. Huddleston J. Structural variant call data. Accession nstd137. dbVar; 2016. https://www.ncbi.n/m.nih.gov/dbvar/studies/nstd137/.

41. Huddleston J, Chaisson M, Steinberg K, Warren W, Hoekzema K, Gordon D, et al. SMRT-SV: Structural variant and indel caller for PacBio reads. Small indel data. 2017. http://eichlerlab.github.io/pacbio_variant_caller/.

42. Dashnow H, Sadedin S, Halman A. Oshlack/STRetch: STRetch v0.2.0. 2018. https://doi.org/10.5281/zenodo.1313915. Accessed 18 Jul 2018.

43. Dashnow H. STRetch summary statistics from 97 PCR-free whole genomes dataset. 2018. https://doi.org/10.6084/m9.figshare.6830282.
Ready to submit your research? Choose BMC and benefit from:

- fast, convenient online submission

- thorough peer review by experienced researchers in your field

- rapid publication on acceptance

- support for research data, including large and complex data types

- gold Open Access which fosters wider collaboration and increased citations

- maximum visibility for your research: over $100 \mathrm{M}$ website views per year

At $\mathrm{BMC}$, research is always in progress.

Learn more biomedcentral.com/submissions 